\title{
A!
}

This is an electronic reprint of the original article.

This reprint may differ from the original in pagination and typographic detail.

Kuhn, C. C.N.; Hoinka, S.; Herrera, I.; Dyke, P.; Kinnunen, J. J.; Bruun, G. M.; Vale, C. J.

\section{High-Frequency Sound in a Unitary Fermi Gas}

Published in:

Physical Review Letters

DOI:

10.1103/PhysRevLett.124.150401

Published: 17/04/2020

Document Version

Publisher's PDF, also known as Version of record

Please cite the original version:

Kuhn, C. C. N., Hoinka, S., Herrera, I., Dyke, P., Kinnunen, J. J., Bruun, G. M., \& Vale, C. J. (2020). HighFrequency Sound in a Unitary Fermi Gas. Physical Review Letters, 124(15), [150401].

https://doi.org/10.1103/PhysRevLett.124.150401

This material is protected by copyright and other intellectual property rights, and duplication or sale of all or part of any of the repository collections is not permitted, except that material may be duplicated by you for your research use or educational purposes in electronic or print form. You must obtain permission for any other use. Electronic or print copies may not be offered, whether for sale or otherwise to anyone who is not an authorised user. 


\title{
High-Frequency Sound in a Unitary Fermi Gas
}

\author{
C. C. N. Kuhn $\odot,{ }^{1}$ S. Hoinka, ${ }^{1}$ I. Herrera $\odot,{ }^{1}$ P. Dyke, ${ }^{1}$ J. J. Kinnunen, ${ }^{2}$ G. M. Bruun $\odot,{ }^{3,4}$ and C. J. Vale ${ }^{1, *}$ \\ ${ }^{1}$ ARC Centre of Excellence in Future Low-Energy Electronics Technologies, Centre for Quantum and Optical Sciences, \\ Swinburne University of Technology, Melbourne 3122, Australia \\ ${ }^{2}$ Department of Applied Physics, Aalto University, FI-00076 Aalto, Finland \\ ${ }^{3}$ Institut for Fysik og Astronomi, Aarhus Universitet, 8000 Aarhus C, Denmark \\ ${ }^{4}$ Shenzhen Institute for Quantum Science and Engineering and Department of Physics, \\ Southern University of Science and Technology, Shenzhen 518055, China
}

(Received 8 December 2019; accepted 25 February 2020; published 13 April 2020)

\begin{abstract}
We present an experimental and theoretical study of the phonon mode in a unitary Fermi gas. Using two-photon Bragg spectroscopy, we measure excitation spectra at a momentum of approximately half the Fermi momentum, both above and below the superfluid critical temperature $T_{\mathrm{c}}$. Below $T_{\mathrm{c}}$, the dominant excitation is the Bogoliubov-Anderson (BA) phonon mode, driven by gradients in the phase of the superfluid order parameter. The temperature dependence of the BA phonon is consistent with a theoretical model based on the quasiparticle random phase approximation in which the dominant damping mechanism is via collisions with thermally excited quasiparticles. As the temperature is increased above $T_{\mathrm{c}}$, the phonon evolves into a strongly damped collisional mode, accompanied by an abrupt increase in spectral width. Our study reveals strong similarities between sound propagation in the unitary Fermi gas and bosonic liquid helium.
\end{abstract}

DOI: $10.1103 /$ PhysRevLett.124.150401

Elementary excitation spectra provide a quantitative picture of the physical properties of matter. In many-body quantum systems, the lowest-lying excitations are typically collective modes such as sound waves, while at higher energies, single-particle excitations dominate. For systems in the normal phase, two distinct regimes of sound propagation generally exist [1]. When the lifetime $\tau$ of the quasiparticles that comprise the excitation is short compared to the sound frequency $\omega$, i.e., $\omega \tau \ll 1$, local thermodynamic equilibrium can be established, and hydrodynamic (first) sound, driven by pressure gradients, is supported. The sound speed is set by the thermodynamic equation of state, and the damping depends upon the shear viscosity and thermal conductivity [2]. In the opposite limit, $\omega \tau \gg 1$, the system is said to be collisionless, but a stable linearly dispersing collective mode can exist for repulsive interactions due to a mean-field restoring force, known as zero sound [1]. In a superfluid, sound waves are driven by gradients in the phase of the order parameter, as shown by Anderson [3,4] and Bogoliubov [5], and later formalized by Goldstone [6]. At long wavelengths, both the frequency and damping of the Bogoliubov-Anderson (BA) phonon coincide with the hydrodynamic (first) sound mode described by Landau's two-fluid theory $[2,7,8]$. But the BA mode can persist to higher frequencies into the collisionless regime.

Sound modes in strongly interacting gases of ultracold fermions have been investigated both theoretically [9-15] and experimentally [16-21]. The unitary Fermi gas is of particular interest since interactions reach the strongest levels allowed by quantum mechanics for a short-range potential, making it an important testing ground for theories of interacting fermions [22-25]. Recent quantitative studies of first $[21,26]$ and second $[17,27]$ sound propagation have shed light on the transport properties [28,29] and superfluid fraction of a Fermi gas at unitarity. As the wave vector $k$ is increased beyond the hydrodynamic regime (when $k>1 / \lambda_{\text {mfp }}$, where $\lambda_{\text {mfp }}$ is the mean free path between collisions), the dispersion of the BA mode remains approximately linear [12,30], such that the sound speed agrees with the $k \rightarrow 0$ limit. The damping, on the other hand, can show strong departures from hydrodynamic behavior [31]. Similar behaviors have been seen in neutron scattering studies on liquid helium, and these provide a basis for understanding changes in the nature of the elementary excitations across the superfluid transition $[32,33]$.

In this Letter, we study sound propagation in a unitary Fermi gas, at a wave vector of approximately half the Fermi wave vector $k_{F}$, as a function of temperature. Using focused beam Bragg spectroscopy, we map the response of the phonon mode in a region of the cloud with near-homogeneous density [20]. In the superfluid phase, we observe a clear BA phonon mode in good agreement with a quasiparticle random phase approximation (QRPA) theory. The QRPA theory assumes collisionless dynamics but includes damping via scattering from thermally excited fermionic quasiparticles [20,34]. Just above $T_{c}$, we observe strongly damped collisional sound, which evolves towards 
single-particle excitations at higher temperatures. Finally, we identify similarities in the temperature dependence of the excitations in the unitary Fermi gas and bosonic liquid ${ }^{4} \mathrm{He}[33,36,37]$.

The experimental sequence employed here is similar to that used previously to study the low-temperature excitations in a Fermi gas with tunable interactions [20]. Briefly, we cool a gas of ${ }^{6} \mathrm{Li}$ atoms in an equal mixture of the two lowest hyperfine states $\left|F=1 / 2, m_{\mathrm{F}}= \pm 1 / 2\right\rangle$ and control the cloud temperature by varying the endpoint of the evaporation. An external magnetic field is tuned to $832.2 \mathrm{G}$, where the $s$-wave scattering length diverges, $|a| \rightarrow \infty$. At this point, elastic collisions are unitarity limited, and the thermodynamic properties of the gas become universal functions of the temperature and density $[8,38,39]$. The cloud is held in a harmonic trap with typical confinement frequencies $\omega_{(x, y, z)} /(2 \pi)=(120,115,24.5) \mathrm{Hz}$. A single-frequency 1064-nm laser provides $x-y$ confinement, and axial trapping arises from a curvature in the applied magnetic field. The power of the 1064-nm trapping light can be tuned to vary the mean atom density $\bar{n}$ in the Bragg scattered volume [20], which in turn sets the Fermi energy $E_{\mathrm{F}}=\hbar^{2} k_{\mathrm{F}}^{2} /(2 m)$ where $m$ is the mass of a lithium atom and $k_{\mathrm{F}}=\left(3 \pi^{2} \bar{n}\right)^{1 / 3}$.

We use two focused Bragg laser beams with $1 / e^{2}$ radii of $20 \mu \mathrm{m}$, intersecting at an angle $2 \theta=12.9^{\circ} \pm 0.2^{\circ}$, to probe a small volume of the cloud where the density is near uniform. The Bragg lasers create a periodic perturbation in the center of the cloud with $k=(4 \pi / \lambda) \sin \theta$, where $\lambda=670 \mathrm{~nm}$ is approximately $1 \mathrm{THz}$ blue-detuned from the nearest atomic transition. For the atom densities used in subsequent experiments, this corresponds to a relative wave vector in the range $0.5 \lesssim k / k_{\mathrm{F}} \lesssim 0.6$. By scanning the relative frequency of the two Bragg lasers over a range of $\omega /(2 \pi)=0 \rightarrow \pm 15 \mathrm{kHz}$, we map the response of the cloud as a function of the Bragg energy. The Bragg lasers are applied with an approximately Gaussian-shaped time envelope and a full width at half maximum (FWHM) of $600 \mu \mathrm{s}$. Immediately after the Bragg pulse, the optical trap is switched off, and the cloud is allowed to expand for $4 \mathrm{~ms}$ before taking an absorption image. The finite duration and size of the Bragg beams lead to a Fourier-limited spectral resolution of approximately $1.25 \mathrm{kHz}$ FWHM, which is well below the typical Fermi energies, $E_{\mathrm{F}} \approx 11 \mathrm{kHz}$, used in our experiments.

Within linear response, momentum $P$ is imparted to the cloud at a rate proportional to the imaginary part of the density-density response function $\chi^{\prime \prime}(k, \omega)$, where $\chi(k, \omega)$ is given by the Fourier transform of $\chi\left(r-r^{\prime}, t-t^{\prime}\right)=$ $-i \vartheta\left(t-t^{\prime}\right)\left\langle\left[\hat{n}(r, t), \hat{n}\left(r^{\prime}, t^{\prime}\right)\right]\right\rangle[40], \hat{n}(r, t)$ is the density operator at position $r$ and time $t$, and $\vartheta(t)$ is the Heaviside function. After a time of flight, the total momentum transferred, $\Delta P$, leads to a displacement of atoms $\Delta x$ from the Bragg scattered volume proportional to $\Delta P$ [41]. All measured Bragg spectra are normalized via the $f$-sum rule $[1,20,42]$,

$$
\frac{\chi^{\prime \prime}(k, \omega)}{2 \pi n \epsilon_{\mathrm{r}}}=\frac{\Delta x(k, \omega)}{\int d \omega \omega \Delta x(k, \omega)}
$$

where $\epsilon_{r}=\hbar k^{2} /(2 m)$ is the atomic recoil energy.

Figure 1(a) shows a selection of measured spectra at unitarity for a range of temperatures below and above $T_{\mathrm{c}} \simeq 0.17 T_{\mathrm{F}}$. Cloud temperatures were determined by fitting in situ density profiles to the equation of state [43]. Below $T_{c}$, we observe a well-defined phonon mode with a decreasing amplitude and increasing width as the temperature approaches $T_{\mathrm{c}}$. Above $T_{\mathrm{c}}$, the mode is noticeably broader, becoming strongly damped with increasing temperature.
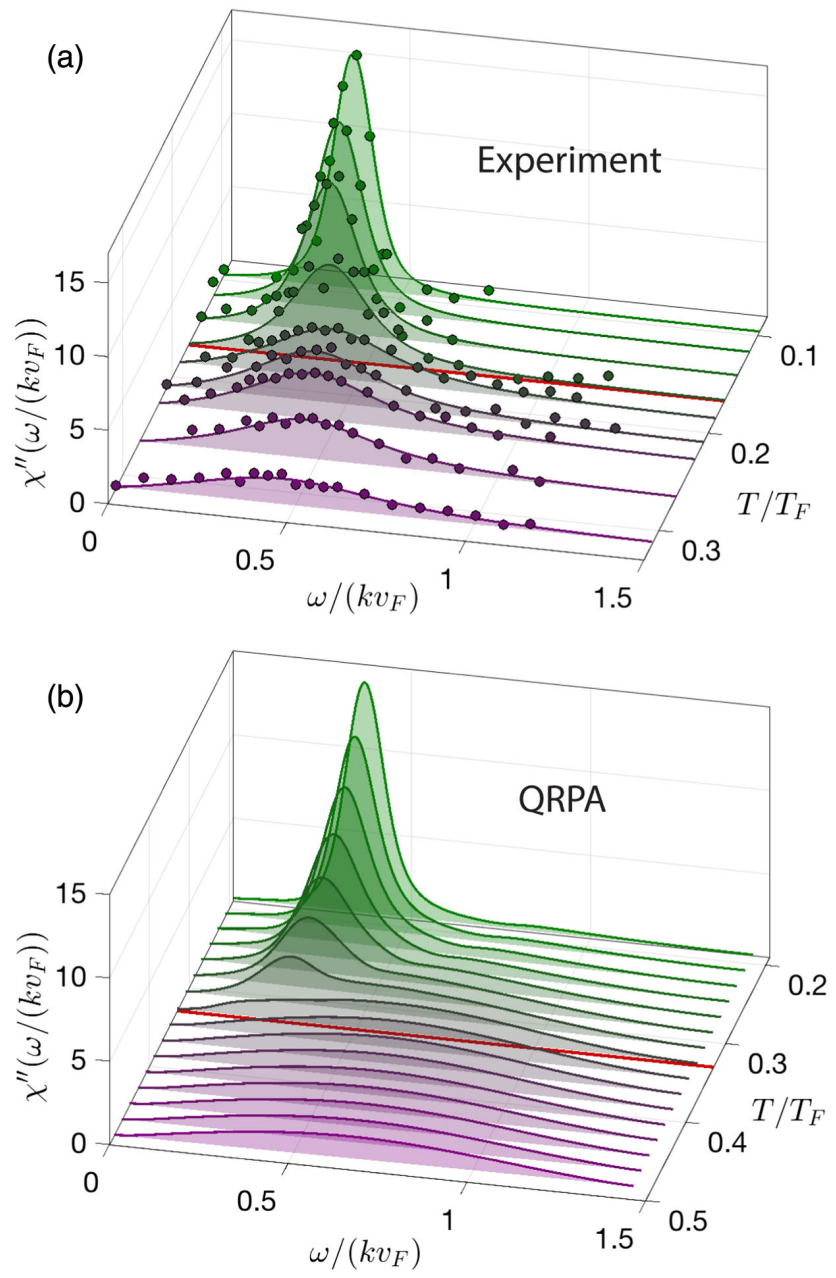

FIG. 1. Excitation spectra for a unitary Fermi gas for a range of temperatures $T / T_{\mathrm{F}}$ at $k / k_{\mathrm{F}}=0.55 \pm 0.03$. (a) Filled circles show the experimental data; solid curves and shaded areas are fits to Eq. (2), convolved with a Gaussian instrumental broadening function as described in the text. (b) Calculated excitation spectra using the QRPA theory, also convolved with the instrumental broadening function. Red solid lines indicate the superfluid transition temperature $T_{c}$. All spectra are presented in units of $\left(4 \pi \epsilon_{\mathrm{r}} E_{\mathrm{F}}\right)^{-1}$. 
Figure 1(b) shows calculated spectra using the QRPA theory $[20,34]$. Note that the QRPA theory yields a different $T_{\mathrm{c}} \sim 0.33 T_{\mathrm{F}}$ to that found in the experiment, but the behavior as a function of $T / T_{\mathrm{c}}$ can be compared. The evolution of the BA phonon mode in the calculated spectra shows good agreement with the experimental data below $T_{\mathrm{c}}$. The theory assumes collisionless dynamics and predicts a well-defined BA mode in the superfluid phase, along with a continuum of single-particle excitations at higher energy but no collective mode in the normal phase due to the absence of zero sound for attractive interactions [1]. Above $T_{\mathrm{c}}$, the QRPA spectra consist of just single-particle excitations.

Inspired by studies of the excitations in liquid helium, we fit our experimental spectra to a damped harmonic oscillator function $[33,36,37]$

$$
\chi^{\prime \prime}(k, \omega)=\frac{Z k \Gamma \omega}{\left(\omega^{2}-\omega_{0}^{2}\right)^{2}+\omega^{2} \Gamma^{2}},
$$

where $\Gamma$ is the damping rate, $\omega_{0}$ is the fundamental frequency of the mode, and $Z$ is a normalization factor. Equation (2) is further convolved with a Gaussian broadening function with FWHM $1.25 \mathrm{kHz}$ before performing the fit, to account for the finite spectral resolution of our measurements. Solid lines and shaded areas in Fig. 1(a) show the fitted functions.

Blue points in Fig. 2(a) show the measured center frequency $\omega_{0}$ of the spectra which, provided the dispersion is approximately linear, serves as a measure of the sound speed. In Fig. 2(b), we check the linearity of the dispersion over a small range of $k / k_{\mathrm{F}}$ at temperatures below and above $T_{\mathrm{c}}, T / T_{\mathrm{F}}=0.11(2)$ (blue circles) and $T / T_{\mathrm{F}}=0.20(2)$ (red squares), respectively. Each point in Fig. 2(b) corresponds to the normalized peak frequency $\hbar \omega_{0} / E_{\mathrm{F}}$ of a Bragg spectrum obtained at a particular density (and hence $\left.k / k_{\mathrm{F}}\right)$. Fitting these data to $\hbar \omega_{0} / E_{\mathrm{F}}=a\left(k / k_{\mathrm{F}}\right)^{b}$, where $a$ and $b$ are fitting parameters, we find $b=1.1 \pm 0.2$ and $1.1 \pm 0.5$, at low and high temperatures, respectively, indicating that the observed dispersion is linear (sonic) within our experimental resolution [12,30,44].

Also shown in Fig. 2(a) are the mode frequencies from the QRPA calculation (grey solid line) and the hydrodynamic sound speed obtained from the thermodynamic equation of state [43] using $c_{\mathrm{s}}^{2}=(\partial \mathcal{P} / \partial \rho)_{s}$, where $\mathcal{P}$ is the pressure, $\rho=n m$ is the mass density, and $s$ is the entropy density (red dash-dotted line). The experimental data for the sound speed are consistent with both the hydrodynamic result and the QRPA theory for $T<T_{\mathrm{c}}$. As $T$ approaches $T_{\mathrm{c}}$ from below, the QRPA theory predicts a downward shift of the BA mode frequency, which is "repelled" from the single-particle continuum. The threshold for single-particle excitations is set by twice the superfluid gap $2 \Delta[12,20,34]$, which decreases with temperature. However, this downshift is not visible in the experimental data. Above $T_{\mathrm{c}}$, our data agree well with the hydrodynamic sound speed but lie
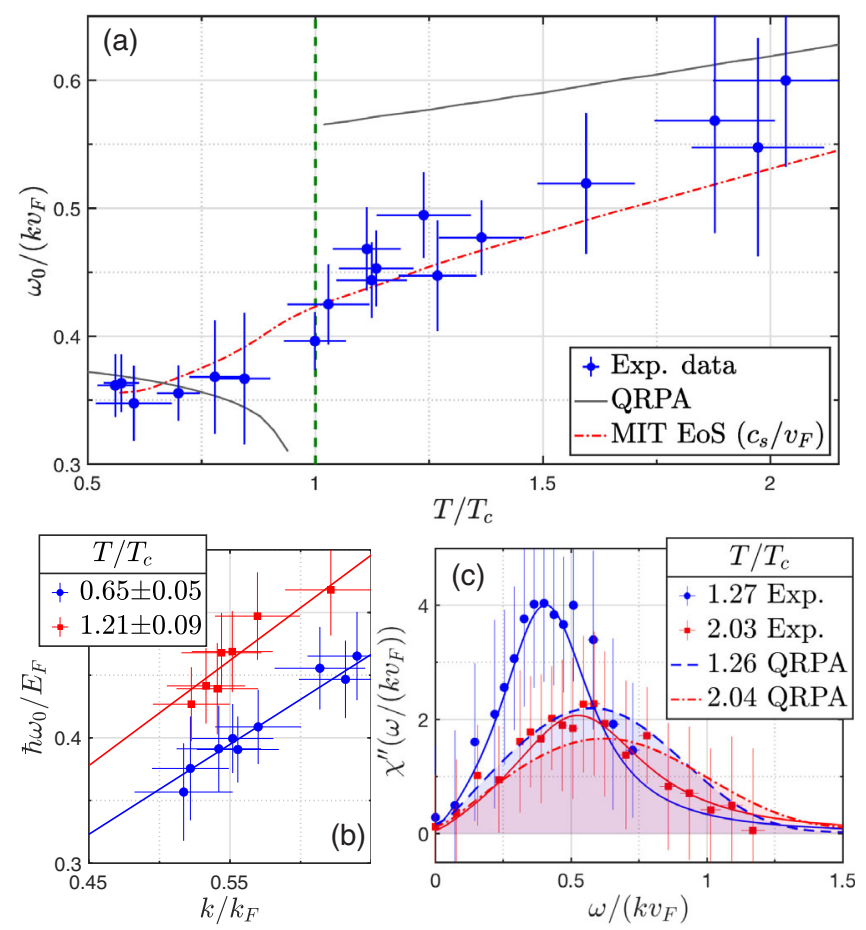

FIG. 2. (a) Mode frequency as a function of $T / T_{\mathrm{c}}$. Blue circles are experimental data, the red dash-dotted line is the hydrodynamic sound frequency [43], and the grey solid line is the QRPA prediction [34]. Note that $T_{\mathrm{c}}$ is indicated by the green dashed line. (b) Mode frequency as a function of momentum at two temperatures, below and above $T_{c}$, respectively. Straight lines are linear fits that cross the origin, confirming the dispersion is linear within experimental resolution. (c) Zoomed-in plots of two experimental spectra (points and solid lines) in the normal phase at two different temperatures along with the corresponding QRPA spectra (dashed and dash-dotted shaded curves). Error bars include uncertainties in the density measurement used to determine $E_{\mathrm{F}}$ and the standard error in the mean of the measured data points.

well below the collisionless single-particle calculation. Figure 2(c) shows a comparison of two sets of experimental and theoretical spectra, in the normal phase at temperatures close to and well above $T_{\mathrm{c}}$. While the theoretical (singleparticle) spectrum at $1.26 T_{\mathrm{c}}$ is very wide, the experimental spectrum at $1.27 T_{\mathrm{c}}$ displays a much narrower peak, indicating the existence of a damped collisional mode in the normal phase. Further above $T_{\mathrm{c}}$, the agreement improves, reflecting the evolution from collective to single-particle excitations at higher temperatures. A Boltzmann equation calculation of the viscous relaxation time $\tau$ supports this result, yielding $0.5 \lesssim \omega_{0} \tau \lesssim 1$ for $T \gtrsim T_{\mathrm{c}}$ [45,46], with $\tau$ growing monotonically with increasing $T$. Similar values of $\omega_{0} \tau$ were obtained using Luttinger-Ward [47] and Gaussian pair fluctuation theories [29]. Our measurements above $T_{\mathrm{c}}$ thus lie in the crossover between the hydrodynamic and collisionless regimes.

In Fig. 3, we plot the amplitude $\max \left[\chi^{\prime \prime}(k, \omega)\right]$, width $\Gamma$, and quality factor $Q=\omega_{0} / \Gamma$ of the phonon mode as a 

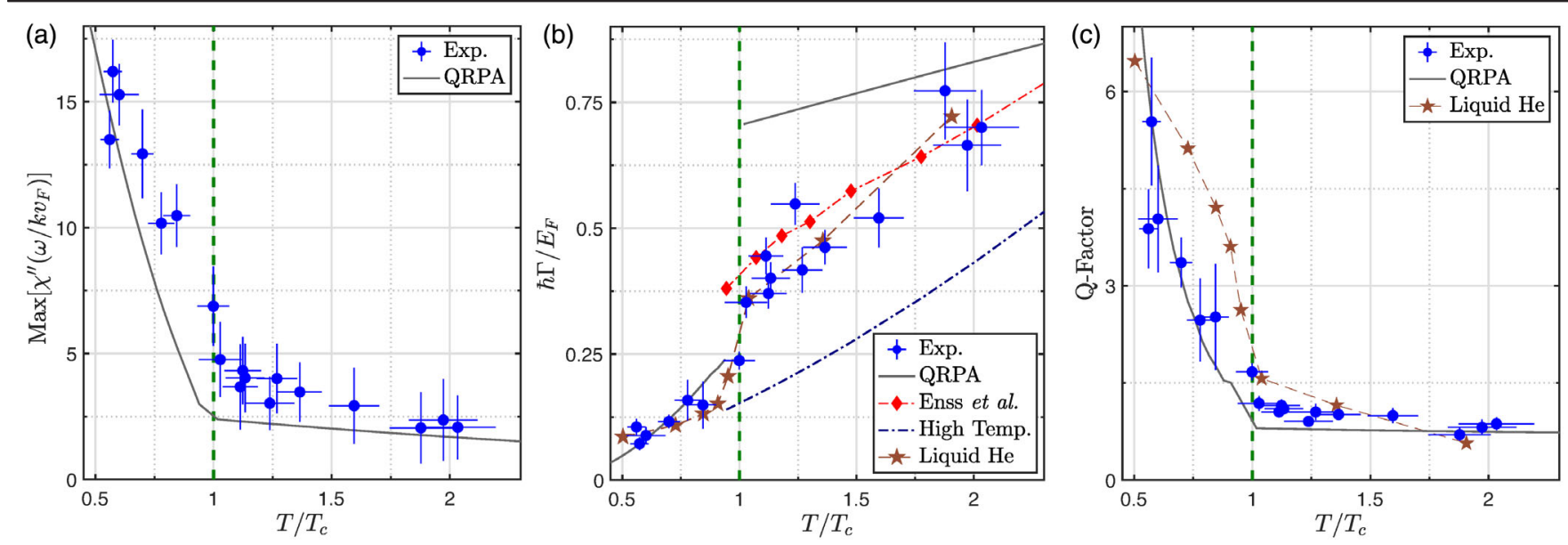

FIG. 3. (a) Normalized peak amplitude of the phonon mode as a function of temperature. Blue circles are experimental data, and the gray solid line is the QRPA calculation. (b) Blue points show the FWHM of the experimental spectra determined by fitting Eq. (2). The grey solid line is the damping predicted by the QRPA theory; blue and red dash-dotted lines are hydrodynamic predictions from Eq. (3) using the classical result for the shear viscosity [46] and for $\eta$ obtained from a $\mathcal{T}$-matrix calculation [47]. (c) Quality factor $\omega_{0} / \Gamma$ of the sound mode. Blue circles are the experimental data; the grey solid line is the QRPA theory. Brown stars and dashed lines in panels (b) and (c) show scaled results for liquid helium measured at a wave vector of $0.4 \AA^{-1}$ [36].

function of the temperature. The amplitude, Fig. 3(a), decreases monotonically with $T$, with a steep gradient below $T_{\mathrm{c}}$ and a shallower gradient above $T_{\mathrm{c}}$. The width, Fig. 3(b), increases with temperature and exhibits a jump at the superfluid transition, reflecting a sudden increase in the damping. These features signify a qualitative change in the sound mode from a BA phonon below $T_{\mathrm{c}}$ to a strongly damped collisional mode in the normal phase.

The QRPA theory accurately describes both the amplitude and width below $T_{\mathrm{c}}$ (Fig. 3). This confirms the collisionless nature of the BA mode at $k \sim k_{\mathrm{F}} / 2$ and identifies collisions with fermionic quasiparticles as the primary damping mechanism. Above $T_{\mathrm{c}}$, the width cannot be described by the collisionless model, nor is it deeply hydrodynamic. To see this, we can compare our data to hydrodynamic theory based on viscous damping. Neglecting the contribution from thermal conductivity (discussed below), the damping rate is given by [8]

$$
\hbar \Gamma / E_{\mathrm{F}}=\frac{8}{3}\left(\frac{k}{k_{\mathrm{F}}}\right)^{2} \frac{\eta}{n}
$$

where $\eta$ is the shear viscosity. The blue dash-dotted curve in Fig. 3(b) is found using Eq. (3) with $\eta=2.77 \hbar n\left(T / T_{\mathrm{F}}\right)^{3 / 2}$, valid in the classical limit $T \gg T_{\mathrm{F}}$ [45,46]. This case is clearly well below the experimental data, which is not surprising given that $T<T_{\mathrm{F}}$. On the other hand, using the shear viscosity calculated from a self-consistent $\mathcal{T}$-matrix approach [47] (red diamonds) yields a damping rate that appears to be consistent with the experimental data. However, this result does not include the damping contribution from thermal conductivity, which should be significant in the hydrodynamic regime $[21,26,48]$.
For $\omega_{0} \tau \sim 1$, there will be too few collisions to enable full rethermalization or momentum relaxation within one period of the sound wave. Therefore, both the viscous and thermal contributions to the damping will be lower than in the hydrodynamic limit as the efficiency of both mechanisms will be reduced, providing further confirmation that our experiments probe the crossover between hydrodynamic and collisionless dynamics.

A recent study of sound propagation in the unitary Fermi gas at long wavelengths, $k \lesssim k_{\mathrm{F}} / 10$, measured the temperature dependence of sound diffusivity $D$ [21]. In the hydrodynamic limit, both the sound frequency $\omega_{0}$ and damping $\Gamma$ were found to evolve smoothly with temperature, including across $T_{\mathrm{c}}$. Below $T_{\mathrm{c}}$, a transition from hydrodynamic damping $\left(\Gamma \propto k^{2}\right)$ to collisionless damping $(\Gamma \propto k)$ was seen with increasing $k$ [21], consistent with a model of sound attenuation in liquid helium [49]. Our data at larger $k$ lie outside the range of hydrodynamic $\left(\Gamma \propto k^{2}\right)$ damping for all reported temperatures. Using the model of Ref. [49], our damping rates below $T_{c}$ connect smoothly to those found in Ref. [21] within experimental uncertainties. The jump in $\Gamma$ we observe at $T_{\mathrm{c}}$ highlights that the BA mode at $k \sim k_{\mathrm{F}} / 2$ is a collisionless excitation driven by phase gradients and that the transition from hydrodynamic to collisionless sound takes place at different $k$ in the superfluid and normal phases.

Finally, we compare the excitations in the unitary Fermi gas to those in liquid helium, which has been studied over several decades [36,50-53]. The brown stars in Fig. 3 show the scaled width (b) and quality factor $\omega_{0} / \Gamma$ (c) of the phonon mode in liquid helium [36], as a function of $T / T_{\mathrm{c}}$. Helium data were obtained via neutron scattering at a wave vector of $k \approx 0.4 \AA^{-1}$, which lies approximately half way 
along the linear (phononic) branch of the dispersion curve, where the excitation energy is approximately half of the roton-gap energy. This case is comparable to the phonon frequency in our measurements being roughly half of the pair-breaking threshold $2 \Delta$. In both systems, this momentum lies in the crossover between the hydrodynamic and collisionless regimes [33]. The qualitative similarity between these two systems as a function of the relative temperature is striking, despite the fact that one system is bosonic and the other is fermionic. This result indicates that interactions dominate the allowed excitations in strongly correlated systems. A similar universality has been observed for the transport coefficients of strongly interacting systems in the hydrodynamic limit across many energy scales spanning cold atomic gases, liquid $\mathrm{He}$, and quarkgluon plasmas $[21,54,55]$. Our results suggest that these similarities extend beyond the hydrodynamic limit.

In conclusion, we have studied sound propagation in a unitary Fermi gas as a function of temperature in the upper part of the phononic branch. The dominant excitation shows a strong temperature dependence and evolves from a collisionless BA mode driven by superfluid phase gradients below $T_{\mathrm{c}}$ to a strongly damped collisional mode above $T_{\mathrm{c}}$. While these different regimes are not distinguished by the sound velocity, the damping rates reveal the differences. Below $T_{\mathrm{c}}$, the damping is dominated by collisions with thermally excited quasiparticles and is well described by a QRPA theory, whereas above $T_{\mathrm{c}}$, the strong damping indicates that the mode lies in the crossover between the collisionless and hydrodynamic regimes. At even higher temperatures, excitation spectra approach the single-particle limit. Finally, we identify strong similarities in the temperature dependence of sound in the unitary Fermi gas and liquid helium at comparable momenta.

We thank M. Zwierlein, Y. Castin, and $\mathrm{H}$. Hu for stimulating discussions and J. Denier for assistance on initial experiments. We acknowledge financial support from the Australian Research Council Program No. CE170100039, and from the Independent Research Fund Denmark-Natural Sciences via Grant No. DFF-802100233B.

*vale@swin.edu.au

[1] D. Pines and P. Nozières, The Theory of Quantum Liquids (Perseus Books Publishing, Benjamin, 1966), Vol. 1.

[2] P. Hohenberg and P. Martin, Ann. Phys. (N.Y.) 34, 291 (1965).

[3] P. W. Anderson, Phys. Rev. 110, 827 (1958).

[4] P. W. Anderson, Phys. Rev. 112, 1900 (1958).

[5] N. N. Bogoljubov, V. V. Tolmachov, and D. V. Širkov, Fortschr. Phys. 6, 605 (1958).

[6] J. Goldstone, Nuovo Cimento (1955-1965) 19, 154 (1961).

[7] L. Landau, Phys. Rev. 60, 356 (1941).
[8] L. Landau and E. Lifshitz, Fluid Mechanics (Elsevier Science, New York, 2013), Vol. 6.

[9] Y. Ohashi and A. Griffin, Phys. Rev. A 67, 063612 (2003).

[10] P. Pieri, L. Pisani, and G. C. Strinati, Phys. Rev. B 70, 094508 (2004).

[11] N. Manini and L. Salasnich, Phys. Rev. A 71, 033625 (2005).

[12] R. Combescot, M. Y. Kagan, and S. Stringari, Phys. Rev. A 74, 042717 (2006).

[13] R. Haussmann, W. Rantner, S. Cerrito, and W. Zwerger, Phys. Rev. A 75, 023610 (2007).

[14] E. Taylor, H. Hu, X.-J. Liu, L. P. Pitaevskii, A. Griffin, and S. Stringari, Phys. Rev. A 80, 053601 (2009).

[15] M. Braby, J. Chao, and T. Schäfer, Phys. Rev. A 82, 033619 (2010).

[16] J. Joseph, B. Clancy, L. Luo, J. Kinast, A. Turlapov, and J. E. Thomas, Phys. Rev. Lett. 98, 170401 (2007).

[17] L. A. Sidorenkov, M. K. Tey, R. Grimm, Y.-H. Hou, L. Pitaevskii, and S. Stringari, Nature (London) 498, 78 (2013).

[18] M. K. Tey, L. A. Sidorenkov, E. R. S. Guajardo, R. Grimm, M. J. H. Ku, M. W. Zwierlein, Y.-H. Hou, L. Pitaevskii, and S. Stringari, Phys. Rev. Lett. 110, 055303 (2013).

[19] W. Weimer, K. Morgener, V. P. Singh, J. Siegl, K. Hueck, N. Luick, L. Mathey, and H. Moritz, Phys. Rev. Lett. 114, 095301 (2015).

[20] S. Hoinka, P. Dyke, M. G. Lingham, J. J. Kinnunen, G. M. Bruun, and C. J. Vale, Nat. Phys. 13, 943 (2017).

[21] P. B. Patel, Z. Yan, B. Mukherjee, R. J. Fletcher, J. Struck, and M. W. Zwierlein, arXiv:1909.02555.

[22] The BCS-BEC Crossover and the Unitary Fermi Gas, edited by W. Zwerger (Springer, Berlin, Heidelberg, 2012).

[23] M. W. Zwierlein, Superfluidity in ultracold atomic Fermi gases, Novel Superfluids (Oxford University Press, Oxford, 2014), pp. 269-422.

[24] I. Bloch, J. Dalibard, and W. Zwerger, Rev. Mod. Phys. 80, 885 (2008).

[25] W. Ketterle and M. W. Zwierlein, in Proceedings of the International School of Physics "Enrico Fermi," Course CLXIV, edited by M. Inguscio, W. Ketterle, and C. Salomon (IOS Press, Amsterdam, 2008).

[26] L. Baird, X. Wang, S. Roof, and J. E. Thomas, Phys. Rev. Lett. 123, 160402 (2019).

[27] M. W. Zwierlein (private communication).

[28] M. Bluhm, J. Hou, and T. Schäfer, Phys. Rev. Lett. 119, 065302 (2017).

[29] H. Hu, P. Zou, and X.-J. Liu, Phys. Rev. A 97, 023615 (2018).

[30] H. Kurkjian, Y. Castin, and A. Sinatra, Phys. Rev. A 93, 013623 (2016).

[31] H. Kurkjian and J. Tempere, New J. Phys. 19, 113045 (2017).

[32] A. Griffin, P. Griffin, G. Allan, A. Goldman, P. McClintock, and M. Springford, Excitations in a Bose-Condensed Liquid, Cambridge Classical Studies (Cambridge University Press, Cambridge, England, 1993).

[33] H. R. Glyde, Rep. Prog. Phys. 81, 014501 (2017).

[34] See Supplemental Material at http://link.aps.org/ supplemental/10.1103/PhysRevLett.124.150401 for details of the QRPA model, which includes Refs. [10,20,35].

[35] R. Haussmann, M. Punk, and W. Zwerger, Phys. Rev. A 80, 063612 (2009). 
[36] R. A. Cowley and A. D. B. Woods, Can. J. Phys. 49, 177 (1971).

[37] H. R. Glyde, Phys. Rev. B 45, 7321 (1992).

[38] T.-L. Ho, Phys. Rev. Lett. 92, 090402 (2004).

[39] S. Nascimbène, N. Navon, K. J. Jiang, F. Chevy, and C. Salomon, Nature (London) 463, 1057 (2010).

[40] A. Brunello, F. Dalfovo, L. Pitaevskii, S. Stringari, and F. Zambelli, Phys. Rev. A 64, 063614 (2001).

[41] G. Veeravalli, E. Kuhnle, P. Dyke, and C. J. Vale, Phys. Rev. Lett. 101, 250403 (2008).

[42] E. D. Kuhnle, H. Hu, X.-J. Liu, P. Dyke, M. Mark, P. D. Drummond, P. Hannaford, and C. J. Vale, Phys. Rev. Lett. 105, 070402 (2010).

[43] M. J. H. Ku, A. T. Sommer, L. W. Cheuk, and M. W. Zwierlein, Science 335, 563 (2012).

[44] G. Bighin, L. Salasnich, P. A. Marchetti, and F. Toigo, Phys. Rev. A 92, 023638 (2015).

[45] P. Massignan, G. M. Bruun, and H. Smith, Phys. Rev. A 71, 033607 (2005).
[46] G. M. Bruun and H. Smith, Phys. Rev. A 72, 043605 (2005).

[47] T. Enss, R. Haussmann, and W. Zwerger, Ann. Phys. (Amsterdam) 326, 770 (2011).

[48] M. Braby, J. Chao, and T. Schäfer, Phys. Rev. A 82, 033619 (2010).

[49] C. Pethick and D. T. Haar, Physica (Utrecht) 32, 1905 (1966).

[50] G. Winterling, F. S. Holmes, and T. J. Greytak, Phys. Rev. Lett. 30, 427 (1973).

[51] J. A. Tarvin, F. Vidal, and T. J. Greytak, Phys. Rev. B 15, 4193 (1977).

[52] J. Maza, F. Miguelez, A. Veira, and F. Vidal, J. Phys. C 21, L75 (1988).

[53] W. G. Stirling and H. R. Glyde, Phys. Rev. B 41, 4224 (1990).

[54] T. Schäfer and D. Teaney, Rep. Prog. Phys. 72, 126001 (2009).

[55] Y. Castin, A. Sinatra, and H. Kurkjian, Phys. Rev. Lett. 119, 260402 (2017). 